\title{
A NOTE ON SKEW LIE PRODUCT OF PRIME RING WITH INVOLUTION
}

\author{
ADNAN ABBASI, MUZIBUR RAHMAN MOZUMDER, AND NADEEM AHMAD DAR
}

Received 05 June, 2018

\begin{abstract}
Let $R$ be a ring with involution. The skew Lie product of $a, b \in R$ is defined by $\nabla[a, b]=$ $a b-b a^{*}$. In the present paper we study prime ring with involution satisfying identities involving skew Lie product and left centralizers.
\end{abstract}

2010 Mathematics Subject Classification: 16W10; 16N60; 16W25

Keywords: prime ring, left centralizer, involution, skew Lie product

\section{INTRODUCTION}

Through out this paper $R$ will be a prime ring with involution $*, Q_{m} r$ and $C$ denotes the maximal right ring of quotient and the extended centroid of $R, H(R)$ will be the set of hermitian elements and $S(R)$ will be the set of skew hermitian elements of $R$. If $\operatorname{char}(R) \neq 2$, involution $*$ is said to be of the first kind if $Z(R) \subset H(R)$, otherwise it is said to be of second kind. In the second case $S(R) \cap Z(R) \neq(0)$. We refer reader to $[6,13]$ for justification and amplification for the above mentioned notations and key definitions.

Following [22], an additive mapping $T: R \rightarrow R$ is said to be a left (resp. right) centralizer (multiplier) of $R$ if $T(x y)=T(x) y$ (resp. $T(x y)=x T(y)$ ) for all $x, y \in R$. If $T$ is both left as well as the right centralizer of $R$, it is said to be the centralizer of $R$. Considerable work has been done on left (resp. right) centralizers (multipliers) in prime and semiprime rings during the last few decades (see for example [2,5, 12, 17, 21, 22]) where further references can be found. The relationship between the commutativity of the ring $R$ and certain specific types of maps on $R$ has been extensively studied over the last few decades. The first result in this direction is due to Divinsky [11], who proved that a simple artinian ring is commutative if it has a commuting non-trivial automorphism. Further refinements and extension has been done by a number of authors in various directions (viz., [1,3,4,7-10,14, 18]). Recently, Ali and Dar [2] proved that if a prime ring with involution of $\operatorname{char}(R) \neq 2$ admits a left centralizer $T: R \rightarrow R$ satisfying any one of the following conditions: (i) 
$T\left(\left[x, x^{*}\right]\right)=0$ (ii) $T\left(x o x^{*}\right)=0$ (iii) $T\left(\left[x, x^{*}\right]\right) \pm\left[x, x^{*}\right]=0$ (iv) $T\left(x o x^{*}\right) \pm\left(x o x^{*}\right)=0$ for all $x \in R$, then $R$ is commutative.

Let $R$ be a ring with an involution $*$. For $a, b \in R$, denote by $\nabla[a, b]=a b-b a^{*}$ the skew Lie product. This kind of product is found playing a more and more important role in some research topics such as representing quadratic functionals with sesquilinear functionals, and its study has attracted many authors attention (see [19,20] and the references therein). Motivated by the theory of rings (and algebras) equipped with a Lie product or a Jordan product, Molnár [15] initiated the systematic study of this skew Lie product, and studied the relation between subspaces and ideals of $B(H)$, the algebra of all bounded linear operators acting on a Hilbert space $H$. Here, an additive map $f$ on $R$ is skew centralizing if $\nabla[a, f(a)] \in Z(R)$ holds for all $a \in R$. The purpose here is to study the skew centralizing left centralizer on prime ring with involution. Moreover some other results involving skew Lie product with respect to left centralizers in prime rings with involution have also been studied.

We shall restrict our attention on left centralizers, since all results presented in this article are also true for right centralizers because of left-right symmetry.

\section{Results}

We begin with the following lemmas, which are essential to prove our main results.

Lemma 1 ([16]). Let $R$ be a prime ring with involution of the second kind. Then $\left[x, x^{*}\right] \in Z(R)$ for all $x \in R$ if and only if $R$ is commutative.

Lemma 2 ([16]). Let $R$ be a prime ring with involution of the second kind. Then $x \circ x^{*} \in Z(R)$ for all $x \in R$ if and only if $R$ is commutative.

Lemma 3 ([13]). Suppose that the elements $a_{i}, b_{i}$ in the central closure of a prime ring $R$ satisfy $\sum a_{i} x b_{i}=0$ for all $x \in R$. If $b_{i} \neq 0$ for some $i$, then $a_{i}^{\prime}$ s are $C$-independent.

Lemma 4. Let $R$ be a prime ring with involution of the second kind such that $\operatorname{char}(R) \neq 2$. If $\nabla\left[x, x^{*}\right] \in Z(R)$ for all $x \in R$, then $R$ is commutative.

Proof. We have $\nabla\left[x, x^{*}\right] \in Z(R)$ for all $x \in R$. On linearizing, we get

$$
\nabla\left[x, y^{*}\right]+\nabla\left[y, x^{*}\right] \in Z(R) \text { for all } x, y \in R .
$$

Replacing $y$ by $k y$, where $k \in S(R) \cap Z(R)$ in (2.1) and using (2.1), we obtain $2 y x^{*} k \in$ $Z(R)$ for all $x, y \in R$ and $k \in S(R) \cap Z(R)$. Replacing $x$ by $x^{*}$ and $y$ by $h \in H(R) \cap$ $Z(R)$, we get $2 x h k \in Z(R)$ for all $x \in R, h \in H(R) \cap Z(R)$ and $k \in S(R) \cap Z(R)$. Since $\operatorname{char}(R) \neq 2$ and $S(R) \cap Z(R) \neq(0)$, this implies that $x \in Z(R)$ for all $x \in R$. That is, $R$ is commutative.

Lemma 5. Let $R$ be a prime ring with involution of the second kind such that $\operatorname{char}(R) \neq 2$. If $R$ admits a nonzero left centralizer $T: R \rightarrow R$ such that $T(x) \circ x^{*} \in$ $Z(R)$ for all $x \in R$, then $R$ is commutative. 
Proof. By the given hypothesis we have

$$
T(x) \circ x^{*} \in Z(R) \text { for all } x \in R .
$$

Linearizing (2.2), we get

$$
T(x) \circ y^{*}+T(y) \circ x^{*} \in Z(R) \text { for all } x, y \in R .
$$

Replacing $y$ by $k y$ in (2.3) and use (2.3), we get

$$
2\left(T(y) \circ x^{*}\right) k \in Z(R) \text { for all } x, y \in R \text { and } k \in S(R) \cap Z(R) .
$$

Since $\operatorname{char}(R) \neq 2$ and $S(R) \cap Z(R) \neq(0)$, this implies that $T(y) \circ x^{*} \in Z(R)$ for all $x, y \in R$. Replacing $x$ by $h$, where $h \in H(R) \cap Z(R)$, we get $2 T(y) h \in Z(R)$ for all $y \in R$ and $h \in H(R) \cap Z(R)$. Since $\operatorname{char}(R) \neq 2$ and $S(R) \cap Z(R) \neq(0)$, we get $T(y) \in R$. This can be further written as $[T(y), r]=0$ for all $y, r \in R$. Replacing $y$ by $y w$ and using the last relation, since $T \neq 0$, we get $R$ is commutative.

Theorem 1. Let $R$ be a prime ring with involution of the second kind such that char $(R) \neq 2$. If $R$ admits a non zero left centralizer $T: R \rightarrow R$ such that $\nabla\left[x, T\left(x^{*}\right)\right] \in Z(R)$ for all $x \in R$, then $R$ is commutative.

Proof. By the given hypothesis

$$
\nabla\left[x, T\left(x^{*}\right)\right] \in Z(R) \text { for all } x \in R .
$$

Linearizing (2.5), we get

$$
\nabla\left[x, T\left(y^{*}\right)\right]+\nabla\left[y, T\left(x^{*}\right)\right] \in Z(R) \text { for all } x, y \in R .
$$

That is,

$$
x T\left(y^{*}\right)-T\left(y^{*}\right) x^{*}+y T\left(x^{*}\right)-T\left(x^{*}\right) y^{*} \in Z(R) \text { for all } x, y \in R .
$$

This further implies that

$$
\left[x T\left(y^{*}\right), r\right]-\left[T\left(y^{*}\right) x^{*}, r\right]+\left[y T\left(x^{*}\right), r\right]-\left[T\left(x^{*}\right) y^{*}, r\right]=0 \text { for all } x, y, r \in R .
$$

Thus

$$
\begin{gathered}
x\left[T\left(y^{*}\right), r\right]+[x, r] T\left(y^{*}\right)-T\left(y^{*}\right)\left[x^{*}, r\right]-\left[T\left(y^{*}\right), r\right] x^{*}+y\left[T\left(x^{*}\right), r\right]+[y, r] T\left(x^{*}\right) \\
-T\left(x^{*}\right)\left[y^{*}, r\right]-\left[T\left(x^{*}\right), r\right] y^{*}=0 \text { for all } x, y, r \in R .
\end{gathered}
$$

Replacing $y$ by $k y$ in (2.7), where $k \in S(R) \cap Z(R)$ and using (2.7), we get

$$
2\left(y\left[T\left(x^{*}\right), r\right]+[y, r] T\left(x^{*}\right)\right) k=0 \text { for all } x, y, r \in R \text { and } k \in S(R) \cap Z(R) .
$$

Since $\operatorname{char}(R) \neq 2$ and $S(R) \cap Z(R) \neq(0)$, this implies that

$$
y\left[T\left(x^{*}\right), r\right]+[y, r] T\left(x^{*}\right)=0 \text { for all } x, y, r \in R .
$$

Taking $y=z$, where $z \in Z(R)$, we get $\left[T\left(x^{*}\right), r\right] z=0$. Then by the primeness of $R$ and the fact that $S(R) \cap Z(R) \neq(0)$, we have $\left[T\left(x^{*}\right), r\right]=0$ for all $x, r \in R$. Taking $x=x^{*}$, we obtain

$$
[T(x), r]=0 \text { for all } x, r \in R .
$$


Replacing $x$ by $x y$ in (2.8), where $y \in R$ and using (2.8), we get $T(x)[y, r]=0$ for all $x, y, r \in R$. Replacing $x$ by $x w$, where $w \in R$. We get $T(x) w[y, r]=0$ for all $x, y, w, r \in R$. Thus by the primeness of $R$, we have either $T(x)=0$ for all $x \in R$ or $[y, r]=0$ for all $y, r \in R$. Since $T \neq 0$, we get $R$ is commutative.

Theorem 2. Let $R$ be a prime ring with involution of the second kind such that char $(R) \neq 2$. If $R$ admits a non zero left centralizer $T: R \rightarrow R$ such that $T\left(\nabla\left[x, x^{*}\right]\right) \in Z(R)$ for all $x \in R$, then $R$ is commutative.

Proof. We have

$$
T\left(\nabla\left[x, x^{*}\right]\right) \in Z(R) \text { for all } x \in R .
$$

Linearizing (2.9), we get

$$
T\left(\nabla\left[x, y^{*}\right]\right)+T\left(\nabla\left[y, x^{*}\right]\right) \in Z(R) \text { for all } x, y \in R .
$$

That is,

$$
T\left(x y^{*}-y^{*} x^{*}\right)+T\left(y x^{*}-x^{*} y^{*}\right) \in Z(R) \text { for all } x, y \in R .
$$

This can be further written as

$$
\begin{aligned}
& T(x)\left[y^{*}, r\right]+[T(x), r] y^{*}-T\left(y^{*}\right)\left[x^{*}, r\right]-\left[T\left(y^{*}\right), r\right] x^{*}+T(y)\left[x^{*}, r\right] \\
+ & {[T(y), r] x^{*}-T\left(x^{*}\right)\left[y^{*}, r\right]-\left[T\left(x^{*}\right), r\right] y^{*}=0 \text { for all } x, y, r \in R . }
\end{aligned}
$$

Replacing $y$ by $k y$ in (2.11) where $k \in S(R) \cap Z(R)$ and using (2.11), we get

$$
2\left(T(y)\left[x^{*}, r\right]+[T(y), r] x^{*}\right) k=0 \text { for all } x, y, r \in R \text { and } k \in S(R) \cap Z(R) .
$$

Since $\operatorname{char}(R) \neq 2$ and $S(R) \cap Z(R) \neq(0)$, we arrive at

$$
T(y)\left[x^{*}, r\right]+[T(y), r] x^{*}=0 \text { for all } x, y, r \in R .
$$

Taking $x=h$, where $h \in H(R) \cap Z(R)$, we get $[T(y), r]=0$ for all $y, r \in R$. Since $S(R) \cap Z(R) \neq(0)$, primeness of $R$ implies that $[T(y), r]=0$ for all $y, r \in R$ which is same as (2.8). Thus proceeding as we did in the Theorem 1, we get the required result.

Theorem 3. Let $R$ be a prime ring with involution of the second kind such that char $(R) \neq 2$. If $R$ admits a left centralizer $T: R \rightarrow R$ such that $\nabla\left[x, T\left(x^{*}\right)\right] \pm \nabla\left[x, x^{*}\right] \in$ $Z(R)$ for all $x \in R$, then either $T$ is centralizer or $R$ is commutative.

Proof. We have

$$
\nabla\left[x, T\left(x^{*}\right)\right] \pm \nabla\left[x, x^{*}\right] \in Z(R) \text { for all } x \in R .
$$

If $T=0$, then by Lemma 4, we get $R$ is commutative. Now consider $T \neq 0$. On linearizing (2.12), we get

$$
\nabla\left[x, T\left(y^{*}\right)\right]+\nabla\left[y, T\left(x^{*}\right)\right] \pm \nabla\left[x, y^{*}\right] \pm \nabla\left[y, x^{*}\right] \in Z(R) \text { for all } x, y \in R .
$$

Replacing $y$ by $k y$ in (2.13), where $k \in S(R) \cap Z(R)$, we have

$$
-*\left[x, T\left(y^{*}\right)\right] k+y T\left(x^{*}\right) k+T\left(x^{*}\right) y^{*} k \mp *\left[x, y^{*}\right] k \pm y x^{*} k \pm x^{*} y^{*} k \in Z(R)
$$


for all $x, y \in R$ and $k \in S(R) \cap Z(R)$. Making use of (2.13), we get $2\left(y T\left(x^{*}\right) \pm y x^{*}\right) k \in Z(R)$ for all $x, y \in R$ and $k \in S(R) \cap Z(R)$. Since $\operatorname{char}(R) \neq 2$ and $S(R) \cap Z(R) \neq(0)$, we obtain $y T\left(x^{*}\right) \pm y x^{*} \in Z(R)$ for all $x, y \in R$. This can be further written as

$$
\left[y T\left(x^{*}\right), r\right] \pm\left[y x^{*}, r\right]=0 \text { for all } x, y, r \in R .
$$

Taking $y=z$ and $x=x^{*}$ and using primeness of $R$ and the fact that $S(R) \cap Z(R) \neq(0)$, we obtain

$$
[T(x), r] \pm[x, r]=0 \text { for all } x, r \in R .
$$

Taking $r=x$, we get

$$
[T(x), x]=0 \text { for all } x \in R
$$

Linearizing (2.14), we have

$$
[T(x), y]+[T(y), x]=0 \text { for all } x, y \in R .
$$

Replacing $x$ by $x w$ in (2.15), where $w \in R$ and using (2.15), we get

$$
T(x)[w, y]+x[T(y), w]=0 \text { for all } x, y, w \in R .
$$

Replacing $y$ by $y m$ in (2.16), where $m \in R$ and using (2.16), we obtain

$$
(T(x) y-x T(y))[w, m]=0 \text { for all } x, y, w, m \in R .
$$

Replacing $m$ by $m u$ where $u \in R$ and using the previous expression, we arrive at

$$
(T(x) y-x T(y)) m[w, u]=0 \text { for all } x, y, w, m, u \in R .
$$

Thus by the primeness of $R$, we have either $T$ is a centralizer or $R$ is commutative.

Theorem 4. Let $R$ be a prime ring with involution of the second kind such that $\operatorname{char}(R) \neq 2$. If $R$ admits a left centralizer $T: R \rightarrow R$ such that $\nabla\left[x, T\left(x^{*}\right)\right] \pm x \circ x^{*} \in$ $Z(R)$ for all $x \in R$, then either $T$ is centralizer or $R$ is commutative.

Proof. We have

$$
\nabla\left[x, T\left(x^{*}\right)\right] \pm x \circ x^{*} \in Z(R) \text { for all } x \in R .
$$

If $T=0$, then by Lemma 2, we have $R$ is commutative. Now consider $T \neq 0$. On linearizing (2.18), we get

$$
\nabla\left[x, T\left(y^{*}\right)\right]+\nabla\left[y, T\left(x^{*}\right)\right] \pm x \circ y^{*} \pm y \circ x^{*} \in Z(R) \text { for all } x, y \in R .
$$

Replacing $y$ by $k y$ in (2.19) where $k \in S(R) \cap Z(R)$, we get

$$
-\nabla\left[x, T\left(y^{*}\right)\right] k+y T\left(x^{*}\right) k+T\left(x^{*}\right) y^{*} k \mp\left(x \circ y^{*}\right) k \pm\left(y \circ x^{*}\right) k \in Z(R)
$$

for all $x, y \in R$ and $k \in S(R) \cap Z(R)$. Making use of (2.19), we obtain

$$
2\left(y T\left(x^{*}\right) \pm y \circ x^{*}\right) k \in Z(R) \text { for all } x, y \in R \text { and } k \in S(R) \cap Z(R) .
$$

Since $\operatorname{char}(R) \neq 2$ and $S(R) \cap Z(R) \neq(0)$, we get

$$
y T\left(x^{*}\right) \pm y \circ x^{*} \in Z(R) \text { for all } x, y \in R .
$$


Taking $x=x^{*}$ and $y=z$, where $z \in S(R) \cap Z(R)$, we obtain

$$
(T(x) \pm 2 x) z \in Z(R) \text { for all } x \in R \text { and } z \in Z(R) .
$$

Using the primeness of $R$, we get $T(x) \pm 2 x \in Z(R)$ for all $x \in R$. This can be further written as $[T(x), r] \pm[2 x, r]=0$ for all $x, r \in R$. Taking $r=x$, we get $[T(x), x] \in R$ for all $x \in R$, which is same as equation (2.14). Thus proceeding on similar lines as in the previous theorem, we get either $T$ is centralizer or $R$ is commutative.

Theorem 5. Let $R$ be a prime ring with involution of the second kind such that $\operatorname{char}(R) \neq 2$. If $R$ admits a left centralizer $T: R \rightarrow R$ such that $T(x) \circ x^{*} \pm \nabla\left[x, x^{*}\right] \in$ $Z(R)$, then either $T$ is centralizer or $R$ is commutative.

Proof. We have

$$
T(x) \circ x^{*} \pm \nabla\left[x, x^{*}\right] \in Z(R) \text { for all } x \in R .
$$

If $T=0$ then by Lemma 4 , we get $R$ is commutative. Now consider $T \neq 0$, linearizing (2.20), we get

$$
T(x) \circ y^{*}+T(y) \circ x^{*} \pm \nabla\left[x, y^{*}\right] \pm \nabla\left[y, x^{*}\right] \in Z(R) \text { for all } x, y \in R .
$$

Replacing $y$ by $y k$ in (2.21), where $k \in S(R) \cap Z(R)$, we get

$$
\left(-T(x) \circ y^{*}\right) k+\left(T(y) \circ x^{*}\right) k \mp \nabla\left[x, y^{*}\right] k \pm y x^{*} k \pm x^{*} y^{*} k \in Z(R)
$$

for all $x, y \in R$. Using (2.21), we have

$$
2\left(T(y) \circ x^{*} \pm y x^{*}\right) k \in Z(R) \text { for all } x, y \in R \text { and } k \in S(R) \cap Z(R) .
$$

Since $\operatorname{char}(R) \neq 2$ and $S(R) \cap Z(R) \neq(0)$, we get

$$
T(y) \circ x^{*} \pm y x^{*} \in Z(R) \text { for all } x, y \in R .
$$

Taking $x=h$, where $h \in H(R) \cap Z(R)$, we get

$$
(2[T(y), r] \pm[y, r]) h \in Z(R) \text { for all } y, r \in R \text { and } h \in H(R) \cap Z(R) .
$$

Since $S(R) \cap Z(R) \neq(0)$, this implies that

$$
2[T(y), r] \pm[y, r] \in Z(R) \text { for all } y, r \in R .
$$

Thus $2[T(y), y]=0$ for all $y \in R$. Since $\operatorname{char}(R) \neq 2$, this implies that $[T(y), y]=0$ for all $y \in R$, which is same as equation (2.14) and the result follows as in the Theorem 3.

Theorem 6. Let $R$ be a prime ring with involution of the second kind such that $\operatorname{char}(R) \neq 2$. If $R$ admits a left centralizer $T: R \rightarrow R$ such that $\nabla\left[x, T\left(x \circ x^{*}\right)\right] \in Z(R)$ for all $x \in R$, then $R$ is commutative. 
Proof. we have

$$
\nabla\left[x, T\left(x \circ x^{*}\right)\right] \in Z(R) \text { for all } x \in R .
$$

Replacing $x$ by $x+y$ in (2.22), we get

$$
\begin{gathered}
x T\left(x \circ y^{*}\right)+x T\left(y \circ x^{*}\right)+x T\left(y \circ y^{*}\right)+y T\left(x \circ x^{*}\right)+y T\left(x \circ y^{*}\right) \\
+y T\left(y \circ x^{*}\right)-T\left(x \circ y^{*}\right) x^{*}-T\left(y \circ x^{*}\right) x^{*}-T\left(y \circ y^{*}\right) x^{*}-T\left(x \circ x^{*}\right) y^{*} \\
-T\left(x \circ y^{*}\right) y^{*}-T\left(y \circ x^{*}\right) y^{*} \in Z(R) \text { for all } x, y \in R .
\end{gathered}
$$

Replace $x$ by $-x$ in (2.23) and adding with (2.23), we get

$$
\begin{aligned}
& 2\left(x T\left(x \circ y^{*}\right)+x T\left(y \circ x^{*}\right)+y T\left(x \circ x^{*}\right)-T\left(x \circ y^{*}\right) x^{*}\right. \\
& \left.-T\left(y \circ x^{*}\right) x^{*}-T\left(x \circ x^{*}\right) y^{*}\right) \in Z(R) \text { for all } x, y \in R .
\end{aligned}
$$

Since $\operatorname{char}(R) \neq 2$, this implies that

$$
\begin{gathered}
x T\left(x \circ y^{*}\right)+x T\left(y \circ x^{*}\right)+y T\left(x \circ x^{*}\right)-T\left(x \circ y^{*}\right) x^{*} \\
-T\left(y \circ x^{*}\right) x^{*}-T\left(x \circ x^{*}\right) y^{*} \in Z(R) \text { for all } x, y \in R .
\end{gathered}
$$

Replacing $y$ by $y k$ in (2.24) and using (2.24), we get

$$
2\left(x T\left(y \circ x^{*}\right)+y T\left(x \circ x^{*}\right)-T\left(y \circ x^{*}\right) x^{*}\right) k \in Z(R)
$$

for all $x, y \in R$ and $k \in S(R) \cap Z(R)$. Since $\operatorname{char}(R) \neq 2$ and $S(R) \cap Z(R) \neq(0)$, this implies that

$$
x T\left(y \circ x^{*}\right)+y T\left(x \circ x^{*}\right)-T\left(y \circ x^{*}\right) x^{*} \in Z(R) \text { for all } x, y \in R .
$$

Replacing $x$ by $k x$ in (2.25), where $k \in S(R) \cap Z(R)$ and using (2.25), we get 2(T(y० $\left.\left.x^{*}\right) x^{*}\right) k^{2} \in Z(R)$ for all $x, y \in R$ and $k \in S(R) \cap Z(R)$. Since $\operatorname{char}(R) \neq 2$ and $S(R) \cap$ $Z(R) \neq(0)$, we arrive at $T\left(y \circ x^{*}\right) x^{*} \in Z(R)$ for all $x, y \in R$. Taking $x=h$, where $h \in H(R) \cap Z(R)$, we get $2 T(y) h^{2} \in Z(R)$ for all $y \in R$ and $h \in H(R) \cap Z(R)$. Since $\operatorname{char}(R) \neq 2$ and $S(R) \cap Z(R) \neq(0)$, this implies that $T(y) \in Z(R)$ for all $y \in R$. This can be further written as $[T(y), r]=0$ for all $y, r \in R$. This is same as equation (2.8). Hence following the same steps as before, we get $R$ is commutative.

Theorem 7. Let $R$ be a prime ring with involution of the second kind such that $\operatorname{char}(R) \neq 2$. If $R$ admits a left centralizer $T: R \rightarrow R$ such that $T\left(\nabla\left[x, x^{*}\right]\right) \pm \nabla\left[x, x^{*}\right] \in$ $Z(R)$ for all $x \in R$, then either $T$ is centralizer or $R$ is commutative.

Proof. We have

$$
T\left(\nabla\left[x, x^{*}\right]\right) \pm \nabla\left[x, x^{*}\right] \in Z(R) \text { for all } x \in R .
$$

If $T=0$, then in view of Lemma 4, we get $R$ is commutative. Now consider $T \neq 0$. Linearizing (2.26), we get

$$
T\left(\nabla\left[x, y^{*}\right]\right)+T\left(\nabla\left[y, x^{*}\right]\right) \pm \nabla\left[x, y^{*}\right] \pm \nabla\left[y, x^{*}\right] \in Z(R) \text { for all } x, y \in R .
$$

Replacing $y$ by $k y$ in (2.27), where $k \in S(R) \cap Z(R)$ and using (2.27), we get

$$
2\left(T(y) x^{*} \pm y x^{*}\right) k \in Z(R) \text { for all } x, y \in R \text { and } k \in S(R) \cap Z(R) \text {. }
$$


Since $\operatorname{char}(R) \neq 2$ and $S(R) \cap Z(R) \neq(0)$, we get

$$
T(y) x^{*} \pm y x^{*} \in Z(R) \text { for all } x, y \in R .
$$

Taking $x=h$, where $h \in H(R) \cap Z(R)$ and using the primeness of $R$ and the fact that $S(R) \cap Z(R) \neq(0)$, we get $T(y) \pm y \in Z(R)$ for all $y \in R$. This can be further written as $[T(y), r] \pm[y, r]=0$ for all $y, r \in R$. Replacing $r$ by $y$, we get $[T(y), y]=0$ for all $y \in R$. Which is same as (2.14). Thus proceeding as before, we get the required result.

Theorem 8. Let $R$ be a prime ring with involution of the second kind such that $\operatorname{char}(R) \neq 2$. If $R$ admits left centralizers $T_{1}, T_{2}: R \rightarrow R$ such that $\left[T_{1}(x), T_{2}\left(x^{*}\right)\right] \pm$ $\nabla\left[x, x^{*}\right] \in Z(R)$ for all $x \in R$, then $R$ is commutative.

Proof. we have

$$
\left[T_{1}(x), T_{2}\left(x^{*}\right)\right] \pm \nabla\left[x, x^{*}\right] \in Z(R) \text { for all } x \in R .
$$

If either $T_{1}=0$ or $T_{2}=0$, then in view of Lemma 4 , we get $R$ be a commutative. Now consider $T_{1} \neq 0$ and $T_{2} \neq 0$, linearizing (2.30), we get

$$
\left[T_{1}(x), T_{2}\left(y^{*}\right)\right]+\left[T_{1}(y), T_{2}\left(x^{*}\right)\right] \pm \nabla\left[x, y^{*}\right] \pm \nabla\left[y, x^{*}\right] \in Z(R) \text { for all } x, y \in R \text {. }
$$

Replacing $y$ by $y k$ in (2.31), where $k \in S(R) \cap Z(R)$ and using (2.31), we get

$$
2\left(\left[T_{1}(y), T_{2}\left(x^{*}\right)\right] \pm y x^{*}\right) k \in Z(R) \text { for all } x, y \in R \text { and } k \in S(R) \cap Z(R) .
$$

Since $\operatorname{char}(R) \neq 2$ and $S(R) \cap Z(R) \neq(0)$, we get

$$
\left[T_{1}(y), T_{2}\left(x^{*}\right)\right] \pm y x^{*} \in Z(R) \text { for all } x, y \in R .
$$

This can be further written as

$$
\left[\left[T_{1}(y), T_{2}\left(x^{*}\right)\right], r\right] \pm\left[y x^{*}, r\right]=0 \text { for all } x, y, r \in R .
$$

Replacing $x$ by $x T_{1}(y)$ in (2.33) and using (2.33), we get

$$
\left(\left[T_{1}(y), T_{2}(x)\right] \pm y x\right)\left[T_{1}(y), r\right]=0 \text { for all } x, y, r \in R .
$$

Replacing $r$ by $r m$ in (2.34) and using (2.34), we get

$$
\left(\left[T_{1}(y), T_{2}(x)\right] \pm y x\right) r\left[T_{1}(y), m\right]=0 \text { for all } x, y, r, m \in R .
$$

Thus by the primeness of $R$, we have either

$$
\left[T_{1}(y), T_{2}(x)\right] \pm y x=0 \text { for all } x, y \in R
$$

or

$$
\left[T_{1}(y), m\right]=0 \text { for all } y, m \in R .
$$

Replace $x$ by $x r$ in (2.36) and using (2.36), we get $T_{2}(x)\left[T_{1}(y), r\right]=0$ for all $x, y, r \in$ $R$. Again replacing $x$ by $x m$, where $m \in R$, we get $T_{2}(x) m\left[T_{1}(y), r\right]=0$ for all $x, y, r, m \in$ $R$. Hence by the primeness of $R$, we get either $T_{2}(x)=0$ for all $x \in R$ or $\left[T_{1}(y), r\right]=0$ for all $y, r \in R$. Since $T_{2} \neq 0$, we have $\left[T_{1}(y), r\right]=0$ for all $y, r \in R$. Replacing $y$ by $y u$, where $u \in R$, we obtain $T_{1}(y)[u, r]=0$ for all $y, u, r \in R$. Replacing $y$ by $y w$, where $w \in R$, we get $T_{1}(y) w[u, r]=0$ for all $y, w, u, r \in R$. Hence by primeness of $R$, we get 
either $T_{1}(y)=0$ for all $y \in R$ or $[u, r]=0$ for all $u, r \in R$. Since $T_{1} \neq 0$, we get $[u, r]=0$ for all $u, r \in R$. That is, $R$ is commutative. Similarly we can get $R$ is commutative in case $\left[T_{1}(y), m\right]=0$ for all $y, m \in R$.

Theorem 9. Let $R$ be a prime ring with involution of the second kind such that char $(R) \neq 2$. If $R$ admit two left centralizers $T_{1}, T_{2}: R \rightarrow R$ such that $\left(\nabla\left[x, x^{*}\right]\right) T_{1}(x) \pm T_{2}(x)\left(\nabla\left[x, x^{*}\right]\right) \in Z(R)$ for all $x \in R$, then either $R$ is commutative or $T_{1}(x)=\mp T_{2}(x)$ for all $x \in R$.

Proof. We have

$$
\left(\nabla\left[x, x^{*}\right]\right) T_{1}(x) \pm T_{2}(x)\left(\nabla\left[x, x^{*}\right]\right) \in Z(R) \text { for all } x \in R .
$$

If $T_{1}=0$ and $T_{2} \neq 0$ then we have

$$
\pm T_{2}(x)\left(\nabla\left[x, x^{*}\right]\right) \in Z(R) \text { for all } x \in R .
$$

This implies that

$$
T_{2}(x)\left(\nabla\left[x, x^{*}\right]\right) \in Z(R) \text { for all } x \in R .
$$

Linearizing (2.40), we get

$$
\begin{gathered}
T_{2}(x)\left(\nabla\left[x, y^{*}\right]\right)+T_{2}(x)\left(\nabla\left[y, x^{*}\right]\right)+T_{2}(x)\left(\nabla\left[y, y^{*}\right]\right)+T_{2}(y)\left(\nabla\left[x, x^{*}\right]\right) \\
+T_{2}(y)\left(\nabla\left[x, y^{*}\right]\right)+T_{2}(y)\left(\nabla\left[y, x^{*}\right]\right) \in Z(R) \text { for all } x, y \in R .
\end{gathered}
$$

Replacing $x$ by $-x$ in (2.41) and using (2.41), we get

$$
2\left(T_{2}(x)\left(\nabla\left[x, y^{*}\right]+T_{2}(x)\left(\nabla\left[y, x^{*}\right]\right)+T_{2}(y)\left(\nabla\left[x, x^{*}\right]\right)\right) \in Z(R) \text { for all } x, y \in R\right. \text {. }
$$

Since $\operatorname{char}(R) \neq 2$, this implies that

$$
T_{2}(x)\left(\nabla\left[x, y^{*}\right]\right)+T_{2}(x)\left(\nabla\left[y, x^{*}\right]\right)+T_{2}(y)\left(\nabla\left[x, x^{*}\right]\right) \in Z(R) \text { for all } x, y \in R \text {. }
$$

Replacing $y$ by $y k$ in (2.43), where $k \in S(R) \cap Z(R)$ and using (2.43), we get

$$
2\left(T_{2}(x) y x^{*}+T_{2}(y)\left(\nabla\left[x, x^{*}\right]\right) k \in Z(R) \text { for all } x, y \in R \text { and } k \in S(R) \cap Z(R)\right. \text {. }
$$

Since $\operatorname{char}(R) \neq 2$ and $S(R) \cap Z(R) \neq(0)$, we get

$$
T_{2}(x) y x^{*}+T_{2}(y)\left(\nabla\left[x, x^{*}\right]\right) \in Z(R) \text { for all } x, y \in R .
$$

This can be further written as

$$
T_{2}(x) y x^{*}+T_{2}(y) x x^{*}+T_{2}(y)\left(x^{*}\right)^{2} \in Z(R) \text { for all } x, y \in R .
$$

Replacing $x$ by $k x$ in (2.45), where $k \in S(R) \cap Z(R)$ and using (2.45), we get

$$
2 T_{2}(y)\left(x^{*}\right)^{2} k \in Z(R) \text { for all } x, y \in R \text { and } k \in S(R) \cap Z(R) .
$$

Since $\operatorname{char}(R) \neq 2$ and $S(R) \cap Z(R) \neq(0)$, this implies that $T_{2}(y)\left(x^{*}\right)^{2} \in Z(R)$ for all $x, y \in R$. Taking $x=h$, where $h \in H(R) \cap Z(R)$, we have $T_{2}(y) h^{2} \in Z(R)$ for all $y \in R$ and $h \in H(R) \cap Z(R)$. Since $S(R) \cap Z(R) \neq(0)$, making use of primeness of $R$, we get $T_{2}(y) \in Z(R)$ for all $y \in R$. This can be further written as $\left[T_{2}(y), r\right]=0$ for all $y, r \in R$. Which is same as equation (2.8). Thus proceeding as in Theorem 1, we have $R$ is commutative. 
Now suppose $T_{2}=0$ and $T_{1} \neq 0$, we get $\left(\nabla\left[x, x^{*}\right]\right) T_{1}(x) \in Z(R)$ for all $x \in R$. Now following the same steps as we follow after (2.40), we get $R$ is commutative. At last consider neither $T_{1} \neq 0$ nor $T_{2} \neq 0$. Linearizing (2.38), we get

$$
\begin{aligned}
& \left(\nabla\left[x, y^{*}\right]\right) T_{1}(x)+\left(\nabla\left[y, x^{*}\right]\right) T_{1}(x)+\left(\nabla\left[y, y^{*}\right]\right) T_{1}(x)+\left(\nabla\left[x, x^{*}\right]\right) T_{1}(y) \\
& +\left(\nabla\left[x, y^{*}\right]\right) T_{1}(y)+\left(\nabla\left[y, x^{*}\right]\right) T_{1}(y) \pm T_{2}(x)\left(\nabla\left[x, y^{*}\right]\right) \pm T_{2}(x)\left(\nabla\left[y, x^{*}\right]\right) \\
& \pm T_{2}(x)\left(\nabla\left[y, y^{*}\right]\right) \pm T_{2}(y)\left(\nabla\left[x, x^{*}\right]\right) \pm T_{2}(y)\left(\nabla\left[x, y^{*}\right]\right) \pm T_{2}(y)\left(\nabla\left[y, x^{*}\right]\right) \\
& \in Z(R) \text { for all } x, y \in R .
\end{aligned}
$$

Replacing $x$ by $-x$ in (2.46) and using (2.46), we get

$$
\begin{gathered}
2\left(\left(\nabla\left[x, y^{*}\right]\right) T_{1}(x)+\left(\nabla\left[y, x^{*}\right]\right) T_{1}(x)+\left(\nabla\left[x, x^{*}\right]\right) T_{1}(y) \pm T_{2}(x)\left(\nabla\left[x, y^{*}\right]\right)\right. \\
\left. \pm T_{2}(x)\left(\nabla\left[y, x^{*}\right]\right) \pm T_{2}(y)\left(\nabla\left[x, x^{*}\right]\right)\right) \in Z(R) \text { for all } x, y \in R .
\end{gathered}
$$

Since $\operatorname{char}(R) \neq 2$, this implies that

$$
\begin{gathered}
\left(\nabla\left[x, y^{*}\right]\right) T_{1}(x)+\left(\nabla\left[y, x^{*}\right]\right) T_{1}(x)+\left(\nabla\left[x, x^{*}\right]\right) T_{1}(y) \pm T_{2}(x)\left(\nabla\left[x, y^{*}\right]\right) \\
\pm T_{2}(x)\left(\nabla\left[y, x^{*}\right]\right) \pm T_{2}(y)\left(\nabla\left[x, x^{*}\right]\right) \in Z(R) \text { for all } x, y \in R .
\end{gathered}
$$

Replacing $x$ by $k x$ in (2.47) and using (2.47), we get

$$
2\left(x y^{*} T_{1}(x)-\left(x^{*}\right)^{2} T_{1}(y) \pm T_{2}(x) x y^{*} \mp T_{2}(y)\left(x^{*}\right)^{2}\right) k^{2} \in Z(R)
$$

for all $x, y \in R$ and $k \in S(R) \cap Z(R)$. As $\operatorname{char}(R) \neq 2$ and $S(R) \cap Z(R) \neq(0)$, we get

$$
x y^{*} T_{1}(x)-\left(x^{*}\right)^{2} T_{1}(y) \pm T_{2}(x) x y^{*} \mp T_{2}(y)\left(x^{*}\right)^{2} \in Z(R) \text { for all } x, y \in R .
$$

Replacing $y$ by $k y$ in (2.48), where $k \in S(R) \cap Z(R)$, and using (2.48), we get

$$
2\left(-\left(x^{*}\right)^{2} T_{1}(y) \mp T_{2}(y)\left(x^{*}\right)^{2}\right) k \in Z(R) \text { for all } x, y \in R \text { and } k \in S(R) \cap Z(R) \text {. }
$$

Since $\operatorname{char}(R) \neq 2$ and $S(R) \cap Z(R) \neq(0)$, this implies that

$$
-\left(x^{*}\right)^{2} T_{1}(y) \mp T_{2}(y)\left(x^{*}\right)^{2} \in Z(R) \text { for all } x, y \in R .
$$

Taking $x=h$, where $h \in H(R) \cap Z(R)$, we get

$$
\left(-T_{1}(y) \pm T_{2}(y)\right) h^{2} \in Z(R) \text { for all } y \in R \text { and } h \in H(R) \cap Z(R) .
$$

Since $S(R) \cap Z(R) \neq(0)$, this implies that

$$
-T_{1}(y) \pm T_{2}(y) \in Z(R) \text { for all } y \in R \text { and } h \in H(R) \cap Z(R) .
$$

If we consider

$$
-T_{1}(y)+T_{2}(y) \in Z(R) \text { for all } y \in R .
$$

This implies

$$
T_{1}(y)-T_{2}(y) \in Z(R) \text { for all } y \in R \text {. }
$$

Hence

$$
\left[T_{1}(y), r\right]-\left[T_{2}(y), r\right]=0 \text { for all } y, r \in R .
$$

Replacing $y$ by $y u$ in (2.49) and using (2.49), we get

$$
\left(T_{1}(y)-T_{2}(y)\right)[u, r]=0 \text { for all } y, u, r \in R .
$$


Thus by the primeness of $R$, we have either $T_{1}(y)=T_{2}(y)$ for all $y \in R$ or $R$ is commutative. Similarly we get either $T_{1}(y)=-T_{2}(y)$ for all $y \in R$ or $R$ is commutative in the case $-T_{1}(y)-T_{2}(y) \in Z(R)$ for all $y \in R$.

Theorem 10. Let $R$ ba a noncommutative prime ring with involution of the second kind such that char $(R) \neq 2$. If $R$ admits two non zero left centralizes $T_{1}, T_{2}: R \rightarrow R$ such that $T_{1}\left(x \circ x^{*}\right) \pm \nabla\left[x, T_{2}\left(x^{*}\right)\right] \in Z(R)$ for all $x \in R$, then $T_{1}=\lambda T_{2}$, where $\lambda \in C$.

Proof. We have

$$
T_{1}\left(x \circ x^{*}\right) \pm \nabla\left[x, T_{2}\left(x^{*}\right)\right] \in Z(R) \text { for all } x \in R .
$$

Linearizing (2.51), we get

$$
T_{1}\left(x \circ y^{*}\right)+T_{2}\left(y \circ x^{*}\right) \pm \nabla\left[x, T_{2}\left(y^{*}\right)\right] \pm \nabla\left[y, T_{2}\left(x^{*}\right)\right] \in Z(R) \text { for all } x, y \in R \text {. }
$$

Replacing $y$ by $k y$ in (2.52), where $k \in S(R) \cap Z(R)$ and using (2.52), we get

$$
2\left(T_{1}\left(y \circ x^{*}\right) \pm y T_{2}\left(x^{*}\right)\right) k \in Z(R) \text { for all } x, y \in R \text { and } k \in S(R) \cap Z(R) .
$$

Since $\operatorname{char}(R) \neq 2$ and $S(R) \cap Z(R) \neq(0)$, this implies that

$$
T_{1}\left(y \circ x^{*}\right) \pm y T_{2}\left(x^{*}\right) \in Z(R) \text { for all } x, y \in R .
$$

Taking $x=x^{*}$ and $y=z$, where $z \in Z(R)$, we obtain

$$
\left(2 T_{1}(x) \pm T_{2}(x)\right) z \in Z(R) \text { for all } x \in R \text { and } z \in Z(R) .
$$

Thus by the primeness and the fact that $S(R) \cap Z(R) \neq(0)$, we have

$$
2 T_{1}(x) \pm T_{2}(x) \in Z(R) \text { for all } x \in R .
$$

This can be further written as

$$
2\left[T_{1}(x), r\right] \pm\left[T_{2}(x), r\right]=0 \text { for all } x, r \in R .
$$

Replacing $r$ by $T_{2}(x)$, we get

$$
2\left[T_{1}(x), T_{2}(x)\right]=0 \text { for all } x \in R .
$$

Since $\operatorname{char}(R) \neq 2$, this implies that

$$
\left[T_{1}(x), T_{2}(x)\right]=0 \text { for all } x \in R .
$$

Linearizing (2.53), we get

$$
\left[T_{1}(x), T_{2}(y)\right]+\left[T_{1}(y), T_{2}(x)\right]=0 \text { for all } x, y \in R .
$$

Replacing $x$ by $x z$ in (2.54) and using (2.54), we get

$$
T_{1}(x)\left[z, T_{2}(y)\right]+T_{2}(x)\left[T_{1}(y), z\right]=0 \text { for all } x, y, z \in R .
$$

Again, replacing $x$ by $x w$ in (2.55) and using (2.55), we get

$$
T_{1}(x) w\left[z, T_{2}(y)\right]+T_{2}(x) w\left[T_{1}(y), z\right]=0 \text { for all } x, y, z, w \in R .
$$


Thus in view of Lemma 3, we have $\left[z, T_{2}(y)\right]=0$ for all $y, z \in R$ or $T_{1}(x)=\lambda(x) T_{2}(x)$, where $\lambda(x) \in C$. But $\left[z, T_{2}(y)\right] \neq 0$ for all $z, y \in R$ since $R$ is not commutative. Hence we get $T_{1}(x)=\lambda(x) T_{2}(x)$, where $\lambda(x) \in C$. Using this in (2.56), we get

$$
\begin{gathered}
\lambda(x) T_{2}(x) w\left[z, T_{2}(y)\right]+T_{2}(x) w\left[\lambda(y) T_{2}(y), z\right]=0 \text { for all } x, y, z, w \in R . \\
\left(\lambda(x) T_{2}(x)-\lambda(y) T_{2}(x)\right) w\left[z, T_{2}(y)\right]=0 \text { for all } x, y, z, w \in R .
\end{gathered}
$$

Using the primeness of $R$ yields that either $\lambda(x) T_{2}(x)-\lambda(y) T_{2}(x)=0$ or $\left[z, T_{2}(y)\right]=0$. Again since $R$ is not commutative we have $\lambda(x) T_{2}(x)=\lambda(y) T_{2}(x)$ and so $T_{1}=\lambda T_{2}$. This completes the proof.

Theorem 11. Let $R$ be a noncommutative prime ring with involution of the second kind such that char $(R) \neq 2$. If $R$ admits two non zero left centralizers $T_{1}, T_{2}: R \rightarrow R$ such that $T_{1}\left(\nabla\left[x, x^{*}\right]\right) \pm \nabla\left[x, T_{2}\left(x^{*}\right)\right] \in Z(R)$, then $T_{1}=\lambda T_{2}$ where $\lambda \in C$.

Proof. We have

$$
T_{1}\left(\nabla\left[x, x^{*}\right]\right) \pm \nabla\left[x, T_{2}\left(x^{*}\right)\right] \in Z(R) \text { for all } x \in R .
$$

Linearizing (2.57), we get

$$
T_{1}\left(\nabla\left[x, y^{*}\right]\right)+T_{1}\left(\nabla\left[y, x^{*}\right]\right) \pm \nabla\left[x, T_{2}\left(y^{*}\right)\right] \pm \nabla\left[y, T_{2}\left(x^{*}\right)\right] \in Z(R) \text { for all } x, y \in R .
$$

Replacing $y$ by $k y$ in (2.58), where $k \in S(R) \cap Z(R)$ and using (2.58), we get

$$
2\left(T_{1}\left(y x^{*}\right) \pm y T_{2}\left(x^{*}\right)\right) k \in Z(R) \text { for all } x, y \in R \text { and } k \in S(R) \cap Z(R) .
$$

Since $\operatorname{char}(R) \neq 2$ and $S(R) \cap Z(R) \neq(0)$, we get

$$
T_{1}\left(y x^{*}\right) \pm y T_{2}\left(x^{*}\right) \in Z(R) \text { for all } x, y \in R .
$$

Taking $y=z$, where $z \in Z(R)$ and $x=x^{*}$, we obtain

$$
\left(T_{1}(x) \pm T_{2}(x)\right) z \in Z(R) \text { for all } x \in R \text { and } z \in Z(R) .
$$

Since $S(R) \cap Z(R) \neq(0)$, we get

$$
T_{1}(x) \pm T_{2}(x) \in Z(R) \text { for all } x \in R .
$$

This can be further written as $\left[T_{1}(x), r\right] \pm\left[T_{2}(x), r\right]=0$ for all $x, r \in R$. Replacing $r$ by $T_{2}(x)$, we get $\left[T_{1}(x), T_{2}(x)\right]=0$ for all $x \in R$. Which is same as equation (2.53), proceeding as before, we get $T_{1}(x)=\lambda T_{2}(x)$ for all $x \in R$.

Theorem 12. Let $R$ be a noncommutative prime ring with involution of the second kind such that char $(R) \neq 2$. If $R$ admits two non zero left centralizers $T_{1}, T_{2}: R \rightarrow R$ such that $\nabla\left[x, T_{1}\left(x^{*}\right)\right] \pm \nabla\left[x, T_{2}\left(x^{*}\right)\right] \in Z(R)$ for all $x \in R$, then $T_{1}=\lambda T_{2}$.

Proof. We have

$$
\nabla\left[x, T_{1}\left(x^{*}\right)\right] \pm \nabla\left[x, T_{2}\left(x^{*}\right)\right] \in Z(R) \text { for all } x \in R .
$$


Linearizing (2.59), we get

$$
\nabla\left[x, T_{1}\left(y^{*}\right)\right]+\nabla\left[y, T_{1}\left(x^{*}\right)\right] \pm \nabla\left[x, T_{2}\left(y^{*}\right)\right] \pm \nabla\left[y, T_{2}\left(x^{*}\right)\right] \in Z(R) \text { for all } x, y \in R .
$$

Replacing $y$ by $k y$ in (2.60) and using (2.60), we get

$$
2\left(y T_{1}\left(x^{*}\right) \pm y T_{2}\left(x^{*}\right)\right) k \in Z(R) \text { for all } x, y \in R \text { and } k \in S(R) \cap Z(R) .
$$

Since $\operatorname{char}(R) \neq 2$ and $S(R) \cap Z(R) \neq(0)$, we get

$$
y T_{1}\left(x^{*}\right) \pm y T_{2}\left(x^{*}\right) \in Z(R) \text { for all } x, y \in R .
$$

Replacing $y$ by $z$, where $z \in Z(R)$ and using primeness, $S(R) \cap Z(R) \neq(0)$ conditions, we obtain $T_{1}\left(x^{*}\right) \pm T_{2}\left(x^{*}\right) \in Z(R)$ for all $x, r \in R$. Taking $x=x^{*}$, we obtain $T_{1}(x) \pm$ $T_{2}(x) \in Z(R)$ for all $x \in R$. This can be further written as $\left[T_{1}(x), r\right] \pm\left[T_{2}(x), r\right]=0$ for all $x \in R$. Replacing $r$ by $T_{2}(x)$, we get $\left[T_{1}(x), T_{2}(x)\right]=0$ for all $x \in R$, which is same as equation (2.53) and hence, we get $T_{1}(x)=\lambda T_{2}(x)$ for all $x \in R$.

Theorem 13. Let $R$ be a prime ring with involution of the second kind such that $\operatorname{char}(R) \neq 2$. If $R$ admits two left centralizers $T_{1}, T_{2}: R \rightarrow R$ such that $T_{1}(x) \circ x^{*} \pm$ $\nabla\left[x, T_{2}\left(x^{*}\right)\right] \in Z(R)$ for all $x \in R$, then $R$ is commutative.

Proof. We have

$$
T_{1}(x) \circ x^{*} \pm \nabla\left[x, T_{2}\left(x^{*}\right)\right] \in Z(R) \text { for all } x \in R .
$$

If $T_{1}=0$ and $T_{2} \neq 0$, then $R$ is commutative by Theorem 1 . If $T_{2}=0$ and $T_{1} \neq 0$, then by Lemma 5, we get $R$ is commutative. Now consider $T_{1} \neq 0$ and $T_{2} \neq 0$, linearizing (2.61), we get

$$
T_{1}(x) \circ y^{*}+T_{1}(y) \circ x^{*} \pm \nabla\left[x, T_{2}\left(y^{*}\right)\right] \pm \nabla\left[y, T_{2}\left(x^{*}\right)\right] \in Z(R) \text { for all } x, y \in R \text {. }
$$

Replacing $y$ by $k y$ in (2.62), where $k \in S(R) \cap Z(R)$ and using (2.62), we get

$$
2\left(T_{1}(y) \circ x^{*} \pm y T_{2}\left(x^{*}\right)\right) k \in Z(R) \text { for all } x, y \in R \text { and } k \in S(R) \cap Z(R) .
$$

Since $\operatorname{char}(R) \neq 2$ and $S(R) \cap Z(R) \neq(0)$, we get

$$
T_{1}(y) \circ x^{*} \pm y T_{2}\left(x^{*}\right) \in Z(R) \text { for all } x, y \in R .
$$

Taking $x=x^{*}$, we obtain

$$
T_{1}(y) \circ x \pm y T_{2}(x) \in Z(R) \text { for all } x, y \in R .
$$

This can be further written as

$$
T_{1}(y)[x, r]+\left[T_{1}(y), r\right] x+x\left[T_{1}(y), r\right]+[x, r] T_{1}(y) \pm y\left[T_{2}(x), r\right] \pm[y, r] T_{2}(x)=0
$$

for all $x, y, r \in R$. Replacing $x$ by $x r$ and using of (2.63), we get

$$
x\left[T_{1}(y), r\right] r+[x, r] T_{1}(y) r+x r\left[T_{1}(y), r\right]+[x, r] r T_{1}(y)=0 \text { for all } x, y, r \in R .
$$

Replacing $x$ by $x+z$ in (2.64) and combining it with (2.64), we obtain

$$
z\left[T_{1}(y), r\right] r+z r\left[T_{1}(y), r\right]=0 \text { for all } y, r \in R \text { and } z \in Z(R) .
$$


Since $S(R) \cap Z(R) \neq(0)$, then primeness of $R$ gives us

$$
\left[T_{1}(y), r\right] r+r\left[T_{1}(y), r\right]=0 \text { for all } y, r \in R .
$$

Substituting $r+z$ for $r$ in (2.65) and using (2.65), we obtain

$$
2\left[T_{1}(y), r\right] z=0 \text { for all } y, z \in R \text { and } z \in Z(R) .
$$

Since $\operatorname{char}(R) \neq 2$ and $S(R) \cap Z(R) \neq(0)$, primeness of $R$ gives $\left[T_{1}(y), r\right]=0$ for all $y, r \in R$. Since $T_{1} \neq 0$, this implies that $R$ is commutative.

Theorem 14. Let $R$ be a noncommutative prime ring with involution of the second kind such that char $(R) \neq 2$. If $R$ admits two left centralizers $T_{1}, T_{2}: R \rightarrow R$ such that $T_{1}\left(\nabla\left[x, x^{*}\right]\right) \pm T_{2}\left(\nabla\left[x, x^{*}\right]\right) \in Z(R)$ for all $x \in R$, then $T_{1}=\lambda T_{2}$ where $\lambda \in C$.

Proof. We have

$$
T_{1}\left(\nabla\left[x, x^{*}\right]\right) \pm T_{2}\left(\nabla\left[x, x^{*}\right]\right) \in Z(R)
$$

If either of $T_{1}=0$ and $T_{2} \neq 0$ or $T_{2}=0$ and $T_{1} \neq 0$, then by Theorem 2, we get $R$ is commutative. Now consider $T_{1} \neq 0$ and $T_{2} \neq 0$, linearizing (2.66), we get

$$
T_{1}\left(\nabla\left[x, y^{*}\right]\right)+T_{1}\left(\nabla\left[y, x^{*}\right]\right) \pm T_{2}\left(\nabla\left[x, y^{*}\right]\right) \pm T_{2}\left(\nabla\left[y, x^{*}\right]\right) \in Z(R) \text { for all } x, y \in R \text {. }
$$

Replacing $y$ by $k y$ in (2.67) and using (2.67), we get

$$
2\left(T_{1}(y) x^{*} \pm T_{2}(y) x^{*}\right) k \in Z(R) \text { for all } x, y \in R \text { and } k \in S(R) \cap Z(R) .
$$

Since $\operatorname{char}(R) \neq 2$ and $S(R) \cap Z(R) \neq(0)$, we get

$$
T_{1}(y) x^{*} \pm T_{2}(y) x^{*} \in Z(R) \text { for all } x, y \in R .
$$

Taking $x=h$, where $h \in H(R) \cap Z(R)$ and using primeness of $R$ and the fact that $S(R) \cap Z(R) \neq(0)$, we get $T_{1}(y) \pm T_{2}(y) \in R$ for all $y \in R$. This can be further written as $\left[T_{1}(y), r\right] \pm\left[T_{2}(y), r\right]=0$ for all $y, r \in R$. Replacing $r$ by $T_{2}(y)$, we get $\left[T_{1}(y), T_{2}(y)\right]=$ 0 for all $y \in R$, which is same as equation (2.53). Hence following the same steps as in Theorem 10, we get $T_{1}(x)=\lambda T_{2}(x)$ for all $x \in R$.

\section{ACKNOWLEDGEMENT}

The authors are very thankful to the referee for his/her careful reading of the paper and valuable suggestions.

\section{REFERENCES}

[1] A. Ali and M. Yasen, "A note on automorphisms of prime and semiprime rings." J. Math. Kyoto Univ., vol. 45, no. 2, pp. 243-246, 2005, doi: 10.1215/kjm/1250281987.

[2] S. Ali and N. Dar, "On centralizers of prime rings with involution." Bull. Iran. Math. Soc., vol. 41, no. 6, pp. 1465-1475, 2015.

[3] S. Ali and N. A. Dar, "On *-centralizing mappings in rings with involution." Georgian Math. J., vol. 21, no. 1, pp. 25-28, 2014, doi: 10.1515/gmj-2014-0006.

[4] M. Ashraf, A. Ali, and S. Ali, "Some commutativity theorems for rings with generalized derivations." Southeast Asian Bull. Math., vol. 31, no. 3, pp. 415-421, 2007. 
[5] M. Ashraf and S. Ali, "On left multipliers and the commutativity of prime rings." Demonstr. Math., vol. 41, no. 4, pp. 763-771, 2008, doi: 10.1515/dema-2008-0404.

[6] K. Beidar, W. Martindale, and A. Mikhalev, Rings with generalized identities. New York, NY: Marcel Dekker, 1996.

[7] H. Bell and W. Martindale, "Centralizing mappings of semiprime rings." Can. Math. Bull., vol. 30, no. 1-3, pp. 92-101, 1987, doi: 10.4153/CMB-1987-014-x.

[8] H. E. Bell and M. N. Daif, "On commutativity and strong commutativity-preserving maps." Can. Math. Bull., vol. 37, no. 4, pp. 443-447, 1994, doi: 10.4153/CMB-1994-064-x.

[9] M. Brešar, "On the distance of the composition of two derivations to the generalized derivations." Glasg. Math. J., vol. 33, no. 1, pp. 89-93, 1991, doi: 10.1017/S0017089500008077.

[10] Q. Deng and M. Ashraf, "On strong commutativity preserving mappings.” Result. Math., vol. 30, no. 3-4, pp. 259-263, 1996, doi: 10.1007/BF03322194.

[11] N. Divinsky, "On commuting automorphisms of rings." Proc. Trans. R. Soc. Canada III (3), vol. 49, pp. 19-22, 1955.

[12] I. R. Hentzel and M. Tammam El-Sayiad, "Left centralizers on rings that are not semiprime." Rocky Mt. J. Math., vol. 41, no. 5, pp. 1471-1482, 2011, doi: 10.1216/RMJ-2011-41-5-1471.

[13] I. Herstein, Rings with involution, Chicago Lectures in Mathematics. The University of Chicago Press, 1976.

[14] T.-K. Lee and T.-L. Wong, "Nonadditive strong commutativity preserving maps." Commun. Algebra, vol. 40, no. 6, pp. 2213-2218, 2012, doi: 10.1080/00927872.2011.578287.

[15] L. Molnár, "A condition for a subspace of $\mathcal{B}(H)$ to be an ideal." Linear Algebra Appl., vol. 235, pp. 229-234, 1996, doi: 10.1016/0024-3795(94)00143-X.

[16] B. Nejjar, A. Kacha, A. Mamouni, and L. Oukhtite, "Commutativity theorems in rings with involution." Commun. Algebra, vol. 45, no. 2, pp. 698-708, 2017, doi: 10.1080/00927872.2016.1172629.

[17] L. Oukhtite, "Left multipliers and Jordan ideals in rings with involution." Afr. Diaspora J. Math., vol. 11, no. 1, pp. 24-28, 2011.

[18] E. C. Posner, "Derivations in prime rings." Proc. Am. Math. Soc., vol. 8, pp. 1093-1100, 1957, doi: $10.2307 / 2032686$.

[19] P. Šemrl, "On Jordan *-derivations and an application.” Colloq. Math., vol. 59, no. 2, pp. 241-251, 1990, doi: 10.4064/cm-59-2-241-251.

[20] P. Šemrl, "Quadratric functionals and Jordan *-derivations.” Stud. Math., vol. 97, no. 3, pp. 157_ $165,1991$.

[21] J. Vukman, "Centralizers on semiprime rings." Comment. Math. Univ. Carolin., vol. 42, no. 2, pp. 237-245, 2001.

[22] B. Zalar, "On centralizers of semiprime rings." Comment. Math. Univ. Carolin., vol. 32, no. 4, pp. 609-614, 1991.

Authors' addresses

Adnan Abbasi

Aligarh Muslim University, Department of Mathematics, Aligarh, India

E-mail address: adnan.abbasi001@gmail.com

Muzibur Rahman Mozumder

Aligarh Muslim University, Department of Mathematics, Aligarh, India

E-mail address: muzibamu81@gmail.com 
Nadeem Ahmad Dar

Islamic University of Science and Technology, Department of Computer Science and Engineering, Awantipora, India

E-mail address: ndmdarlajurahegmail.com 